\title{
Turning Waste Oil to High Standard Commercial Biodiesel
}

\author{
Penjit Srinophakun, Rawinun Junsitiwate, Anusith Thanapimmetha, Methee Saisriyoot \\ Kasetsart University \\ 50 Ngamwongwan Road, Chatuchak, Bangkok, Thailand \\ fengpjs@ku.ac.th; tuk_rose@hotmail.com; fengjrc@ku.ac.th; fengmts@ku.ac.th
}

\section{Extended Abstract}

Biodiesel is renewable energy derived from triglyceride such as vegetable oil or animal fat and alcohol through the chemical and biological reactions. Theoretically, $10 \%$ glycerol, by-product, is produced from the process. This crude glycerol contains triglycerides, alcohols, catalysts, free fatty acid, fatty acid methyl eater and water which can be purified from about 70-80\% to about $95-99.9 \%$ purity. There are two steps of purification, acid hydrolysis and distillation. Acid hydrolysis or acidulation will separate the crude glycerol to 3 layers namely waste oil, glycerol and sulphate salt. The obtained glycerol from the first step will be distilled to get pure glycerol. This paper will present on how to make high standard commercial biodiesel from this waste oil.

Two-step catalysis of esterification and transesterification was applied to produce the biodiesel from the waste oil. For the first step of esterification, the methanol to oil ratio of 14:1 to 28:1 at the constant reaction time of 165 minutes and $0.66 \%$ acid catalyst were performed (Arora et. al., 2016). It was found that at the methanol to oil ratio of 14:1 could reduce the free fatty acid contents in the waste oil from $13.29 \%$ to $0.56 \%$. For the second step of transesterification, the methanol and oil ration of 6:1 to 9:1 and reaction time of 45-90 minutes were varied. The optimum conditions of transesterification were found at the methanol to oil ratio of 9:1 and reaction time of 60 minutes which gave 94.72 wt $\%$ of the fatty acid methyl ester content (FAME) and $0.34 \mathrm{mg} \mathrm{KOH} / \mathrm{g}$ of the acid value.

According to the Thai Commercial Biodiesel Standard, FAME has to be higher than 96.5\%. Therefore, further purification process using adsorption was applied. In this case, $\%$ of activated carbon and absorption time were varied in the range of 1-3 wt $\%$ and 20-60 minutes. The results showed that 97.31\%FAME was obtained at the conditions of 2 wt $\%$ activated carbon and 40 minutes contact time at $60{ }^{\circ} \mathrm{C}$. Nevertheless, at this conditions the viscosity, acid value and oxidation stability of biodiesel were analyzed and were $4.43 \mathrm{cSt}, 0.32 \mathrm{mg} \mathrm{KOH} / \mathrm{g}$, and $20.60 \mathrm{~h}$ (500 ppm of TBHQ added), respectively. It is obviously all fuel properties of produced biodiesel met the quality of commercial standard of biodiesel in Thailand.

\section{References}

[1] M. Chai, Q. Tu, M. Lu and Y. J. Yang., "Esterification pretreatment of free fatty acid in biodiesel production from laboratory to industry," Fuel Processing Technology, vol. 125, pp. 106-13, 2014.

[2] M. Hema, S. Arivoli and B. Rhodamine, "Adsorption by activated carbon: kinetic and equilibrium studies," Indian Journal of Chemical Technology, vol. 16, pp. 38-45, 2009.

[3] M. L. Savaliya, B. D. Dhorajiya and B. Z. Dholakiya, "Current trends in separation and purification of fatty acid methyl ester," Separation \& Purification Reviews, vol. 44, pp. 28-40, 2015.

[4] R. Arora, "Esterification of high free fatty acid rice bran oil: parametric and kinetic study," Chemical and Biochemical Engineering, vol. 29, pp. 617-623, 2016. 